\title{
An Improved Element Based Lattice Reduction Algorithm for Large MIMO Detection
}

\author{
Tatsuki Fukuda ${ }^{\mathrm{a}, *}$, Sota Uchimura ${ }^{\mathrm{a}}$, Kento Fukai ${ }^{\mathrm{b}}$, Hua-An Zhao ${ }^{\mathrm{b}}$ \\ ${ }^{a}$ National Institute of Technology, Kitakyushu College, 5-20-1 Shii, Kokuraminami-ku, Kitakyushu-city, Fukuoka 802- \\ 0985, Japan \\ ${ }^{b}$ Kumamoto University, 2-39-1 Kurokami, Chuo-ku, Kumamoto-city, Kumamoto 860-8555, Japan \\ *Corresponding Author: tfukuda@kct.ac.jp
}

\begin{abstract}
The element based lattice reduction (ELR) is a kind of lattice reduction technique, and it is often used with some linear detection method to make orthogonality higher. The original ELR, however, needs a lot of iterations because the termination condition is not established easily due to the rounding function used in it. We propose a new value using a flooring function. We conducted some experiments, and they show that our proposal have almost the same performance of bit error ratio as the original ELR. The experiments also show that ours needs lower iterations than the original, and ours can improved over $40 \%$ of the number of iterations under the condition of the number of antennas is 128 and signal-to-noise ratio is $30 \mathrm{~dB}$. It means that our proposal is almost the same bit-error-ratio performance with lower complexity than the original.
\end{abstract}

Keywords: Massive MIMO, Detection, Lattice Reduction.

\section{Introduction}

Wireless communication systems employing lots of antennas for both of transmit and receive have attracted many researchers. These systems are called MIMO (Multiple-Input Multiple-Output) wireless communication systems, and can achieve higher date rate and higher reliability communications than SISO (Single-Input SingleOutput) which have one transmit antenna and one receive antenna. The more antennas MIMO wireless systems use, the larger gain MIMO systems can obtain ${ }^{(1-3)}$. Recently, a study on the 5-th generation mobile wireless systems (5G) are proceeding, and massive MIMO systems are expected as a technique which meet a requirement for $5 \mathrm{G}$. A massive
MIMO system employs hundreds of antennas to aim large capacity communication, so a study on MIMO systems is essential for the growth of communication systems ${ }^{(4,5)}$.

MIMO wireless systems, however, have a problem. That's a decrease of accuracy of signal detection at receiver due to interference between signals which are transmitted at the same time and on the same frequency. Moreover the noises added into the signals make signal detection difficult. Because of these factors, the signal detection technique for received signals is so important. A detection method which achieve the best accuracy is a ML (Maximum Likelihood), which is not useful because the computational complexity is exponential function of the number of antennas. On the other hand, ZF (Zero Forcing) method, MMSE (Minimum Mean square Error) method, and other linear detection methods have a low computational complex. They, however, cannot obtain the large gain with a lot of antennas because the accuracy is not better than ML. The massive MIMO systems have a lot of antennas, so they are not optimal.

These days, the detection methods using LR (Lattice Reduction) technique are drawing attention. LR reduction can raise the accuracy of linear detection method to the same of ML with a low computational complexity by increasing orthogonality of channel matrix ${ }^{(6)}$. The LLL (Lenstra Lenstra Lovasz) algorithm is one of the LR technique ${ }^{(7)}$, and LLLaided linear detection can achieve the same diversity as the ML for MIMO systems ${ }^{(8-10)}$. ELR (Element based Lattice Reduction) algorithm is another of LR technique, which is often adopted to the massive MIMO systems ${ }^{(11)}$. ELR algorithm transforms the channel matrix into dual lattice, and the orthogonality increase by minimize the diagonal element. In ELR, the end of recursive lattice reduction process is depend on a variable, and a lot of calculations are needed. In 
this paper, we propose an improved ELR (IELR) algorithm which can reduce the calculation time, and show that the proposed IELR algorithm is almost the same detection accuracy as the ELR.

\section{System Model}

We consider a MIMO system with $N_{\mathrm{T}}$ antennas for transmit and $N_{\mathrm{R}}$ for receive. The received signal matrix $\mathbf{y} \in$ $\mathbb{C}^{N_{R}}$ can be written as $\mathbf{y}=\boldsymbol{H} \mathbf{x}+\mathbf{n}$, where $\mathbf{x} \in \mathbb{C}^{N_{T}}$ denotes a transmitted signal vector whose elements are generated as M-QAM symbols, $\mathbf{H} \in \mathbb{C}^{N_{R} \times N_{T}}$ denotes a channel matrix whose entries are independent, identically distributed complex Gaussian random variables with variance 0.5 in each dimension, $\mathbf{n} \in \mathbb{C}^{N_{R}}$ denotes a noise vector.

\section{ELR Algorithm}

The ELR algorithm is a LR technique for massive MIMO systems, and it can find the reduced lattice from pairwise error ratio. The input of ELR is $\mathbf{C}$ which is a scaled covariance matrix of the noise after equalization and $\mathbf{C}=$ $\left(\boldsymbol{H}^{H} \boldsymbol{H}\right)^{-\mathbf{1}}$ where $\boldsymbol{H}^{H}$ is the Hermitian of $\boldsymbol{H}$. The output of ELR is a unimodular matrix $\boldsymbol{T}$ which satisfied with the equation $\widetilde{\boldsymbol{H}}=\boldsymbol{H T}$, where $\widetilde{\boldsymbol{H}}$ has more orthogonality than $\boldsymbol{H}$ and has the same lattice as $\boldsymbol{H}$. The entries of $\boldsymbol{T}$ and $\boldsymbol{T}^{\mathbf{- 1}}$ are Gaussian integer, and the determinant of $\boldsymbol{T}$ is \pm 1 or $\pm j$.

First, ELR choose a pair of indices $(i, k)$ and calculate $\lambda_{i, k} \in \mathbb{Z}[\mathrm{j}]$, where $\mathbb{Z}[\mathrm{j}]$ denotes the Gaussian integer ring whose elements have a form $\mathbb{Z}+\mathrm{j} \mathbb{Z}$, and then update the $k$ th column of matrix $\boldsymbol{T}^{\prime}=\left(\boldsymbol{T}^{-1}\right)^{H}$ as,

$$
\boldsymbol{t}_{k}^{\prime} \leftarrow \boldsymbol{t}_{k}^{\prime}+\lambda_{i, k} \boldsymbol{t}_{i}^{\prime}
$$

where $\boldsymbol{t}_{i}^{\prime}$ is the $i$-th column of $\boldsymbol{T}^{\prime}$. Also $k$-th column and $k$-th row of $\widetilde{\boldsymbol{C}}=\left(\widetilde{\boldsymbol{H}}^{H} \widetilde{\boldsymbol{H}}\right)^{-1}$, which are named $\tilde{\boldsymbol{c}}_{k}$ and $\tilde{\boldsymbol{c}}^{(k)}$, respectively, are updated as follows,

$$
\begin{gathered}
\tilde{\boldsymbol{c}}_{k} \leftarrow \tilde{\boldsymbol{c}}_{k}+\lambda_{i, k} \tilde{\boldsymbol{c}}_{i} \\
\tilde{\boldsymbol{c}}^{(k)} \leftarrow \tilde{\boldsymbol{c}}^{(k)}+\lambda_{i, k}^{*} \tilde{\boldsymbol{c}}^{(i)},
\end{gathered}
$$

where superscript $*$ denotes the conjugate. Thus, the $\widetilde{\boldsymbol{H}}$ is updated as follows,

$$
\widetilde{\boldsymbol{h}}_{i} \leftarrow \widetilde{\boldsymbol{h}}_{i}-\lambda_{i, k}^{*} \widetilde{\boldsymbol{h}}_{k},
$$

where $\widetilde{\boldsymbol{h}}_{i}$ is the $i$-th column of $\widetilde{\boldsymbol{H}}$. Now, the (k, k)-th entry of $\widetilde{\boldsymbol{C}}$ is updated as

$$
\tilde{C}_{k, k} \leftarrow \tilde{C}_{k, k}+\left|\lambda_{i, k}\right|^{2} \tilde{C}_{i, i}+\lambda_{i, k}^{*} \tilde{C}_{i, k}+\lambda_{i, k} \tilde{C}_{k, i}
$$

so the amount that $\tilde{C}_{k, k}$ decrease is

$$
\Delta_{i, k}=-\left|\lambda_{i, k}\right|^{2} \tilde{C}_{i, i}-\lambda_{i, k}^{*} \tilde{C}_{i, k}-\lambda_{i, k} \tilde{C}_{k, i} .
$$

Note that the diagonal elements except for $\tilde{C}_{k, k}$ is not updated. $\tilde{C}_{k, k}$ is minimized when the following formula is established

$$
\lambda_{i, k}=-\left\lfloor\frac{\tilde{C}_{i, k}}{\tilde{C}_{i, i}}\right\rceil,
$$

where $[\cdot]$ denotes a rounding function, ELR algorithm finish the process when $\lambda_{i, k}=0$, and a unimodular matrix $\boldsymbol{T}$ is obtained. ELR algorithm is shown in Algorithm 1.

ELR algorithms finish the process when all $\lambda_{i, k}=0$ for any $i \neq k$, so there is a problem that $\lambda_{i, k}$ has to be recalculated every iterations and a lot of iterations are needed. We propose an improved ELR algorithm in next section to reduce the number of calculations of $\lambda_{i, k}$.

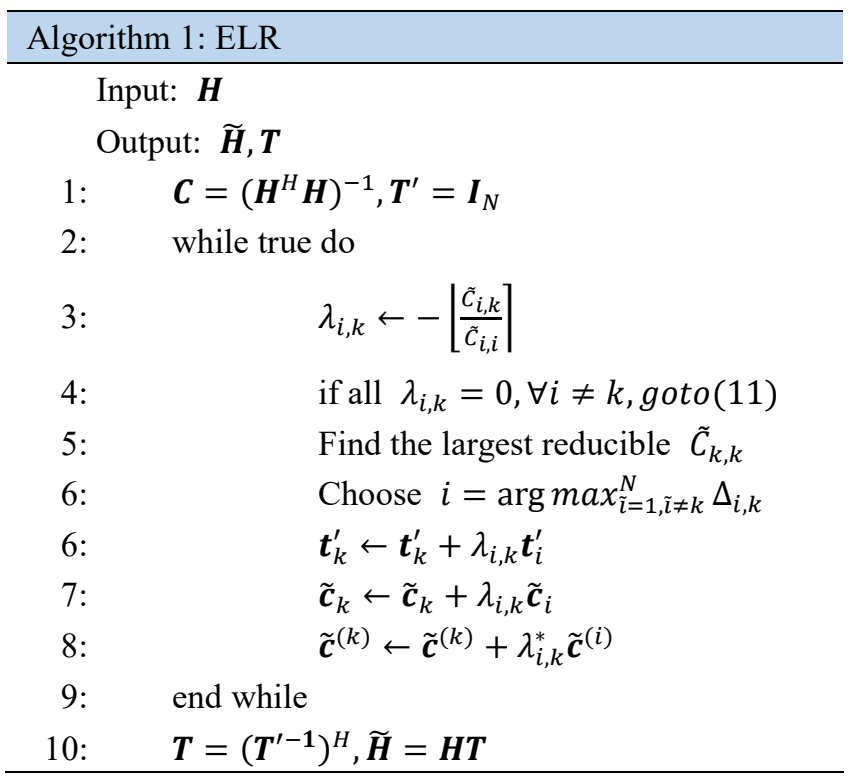

\section{Improvement of ELR Algorithm}

The PEP (Pair-wise Error Probability) can be calculated with $\widetilde{\boldsymbol{C}}$. For example, the PEP of ZF using LR is calculated as follows.

$$
\mathrm{P}\left(z_{i} \rightarrow \tilde{z}_{i} \mid \boldsymbol{H}\right)=\mathrm{A}\left(\sqrt{\frac{\left|e_{z_{i}}\right|^{2}}{2 \sigma_{\omega}^{2} \tilde{C}_{i, i}}}\right)
$$

where $z_{i}, \tilde{z}_{i}, e_{z_{i}}, \sigma_{\omega}^{2}$ denote transmitted symbol, received symbol, difference of them, noise power, respectively, and $\mathrm{A}(x)=(2 \pi)^{-1 / 2} \int_{x}^{\infty} \exp \left(-t^{2} / 2\right) d t$. As you see, PEP decrease when $C_{i, i}$ is decrease. ELR try to minimize $\widetilde{\boldsymbol{C}}$ in order to minimize PEP, but PEP is influenced by $e_{z_{i}}$ and $\sigma_{\omega}^{2}$. It means that PEP becomes small enough 
while $\widetilde{\boldsymbol{C}}$ is not minimized. The number of iterations in conventional ELR algorithm is too large to obtain the enough detection performance. That's because the orthogonality is improved even if not all $\lambda_{i, k}$ become zero. Our idea to improve ELR is termination conditions of process, which is "not all $\lambda_{i, k}$ for any $i \neq k$ become zero, but all $\lambda_{i, k}$ become almost zero."

We propose the new equation to calculate $\lambda_{i, k}$ as follows,

$$
\lambda_{i, k}=- \text { Floor }\left[\frac{\tilde{C}_{i, k}}{\tilde{C}_{i, i}}\right],
$$

where Floor ['] denotes a floor function. With this equation, the $\lambda_{i, k}$ become zero faster, so the number of iterations decrease. BER (Bit Error Ratio), however, decrease if the initial orthogonality is low, so we use both of Equation (6) and (8) depend on a threshold $\alpha$ as follows.

$$
\lambda_{i, k}=\left\{\begin{array}{l}
\left.-\mid \frac{\tilde{C}_{i, k}}{\tilde{C}_{i, i}}\right\rceil \quad\left(\tilde{C}_{i, k}>\alpha\right) \\
- \text { Floor }\left[\frac{\tilde{C}_{i, k}}{\tilde{C}_{i, i}}\right] \quad\left(\tilde{C}_{i, k} \leq \alpha\right)
\end{array}\right.
$$

\section{Threshold for IELR}

To reduce the number of iterations with keeping BER performance, we should use $\lambda_{i, k}$ with rounding function in case of low orthogonality, and $\lambda_{i, k}$ with flooring function in the other case. In Massive MIMO systems, the detection performance will be influenced by noise, which is more than MIMO, due to fading. We assume that minimum orthogonality can be kept if the PEP represented as Equation

(7) is smaller than $10^{-4}$. PEP via $x=\sqrt{\frac{\left|e_{z_{i}}\right|^{2}}{2 \sigma_{\omega}^{2} \tilde{C}_{i, i}}}$ is shown in

Table 1. PEP is about $10^{-4}$ when $x=3.7$. it means that PEP is smaller than $10^{-4}$ when $x$ is larger than 3.7 , so we can obtain the condition of $\tilde{C}_{i, i}$ as follows.

$$
\tilde{C}_{i, i}<\frac{\left|e_{z_{i}}\right|^{2}}{27.38 \sigma_{\omega}^{2}}
$$

Table 1. Pair-wise Error Probability of ELR-ZF.

\begin{tabular}{|c|c|}
\hline$x$ & PEP \\
\hline 1 & $1.59 \times 10^{-1}$ \\
\hline 2 & $2.28 \times 10^{-2}$ \\
\hline 3 & $1.30 \times 10^{-3}$ \\
\hline 4 & $3.17 \times 10^{-5}$ \\
\hline 5 & $2.87 \times 10^{-7}$ \\
\hline
\end{tabular}

Now, from constellation of QAM, we assume that the detector's performance is good if $\left|e_{z_{i}}\right|<\sqrt{2}$ and that the number of antennas and modulation is 128 and 64QAM, respectively. The $\sigma_{\omega}^{2}=0.1450$ when the target SNR is $30 \mathrm{~dB}$. Thus, we obtained the following condition,

$$
\tilde{C}_{i, i}<0.5
$$

So, we use 0.5 as threshold $\alpha$. Our proposed IELR algorithm is shown in Algorithm 2.

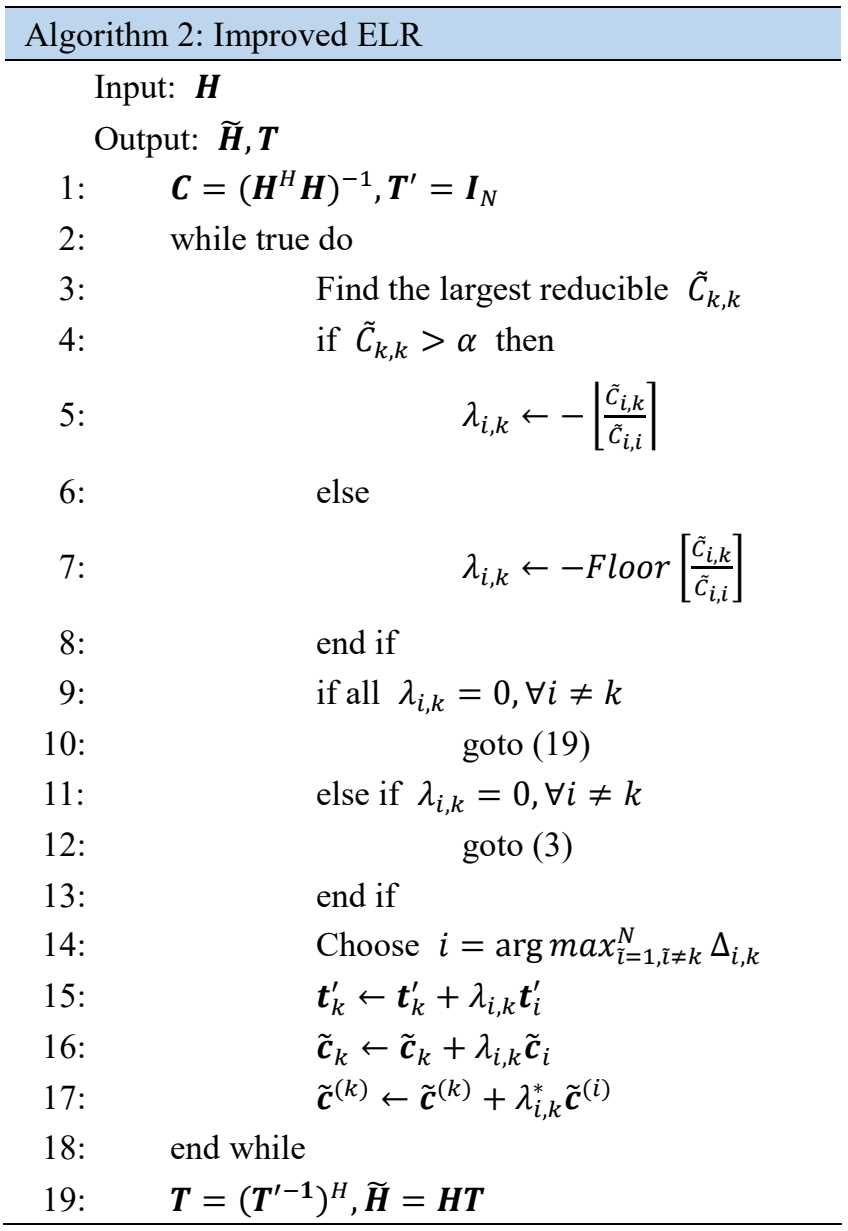

\section{Threshold for IELR}

We conducted some experiments with MATLAB $\mathrm{R} 2012 \mathrm{~b}$ to verify the superiority of our improvement. We assume that the modulation in transmitter is 64 QAM, the channel is adaptive white Gaussian noise, and the receiver has channel information.

\subsection{BER Performance via SNR}

The BER performances of LLL, original ELR, and ours (IELR) are shown in Fig. 1 to Fig. 4. The horizontal axis represents the SNR (Signal-to-Noise Ratio) and the vertical 


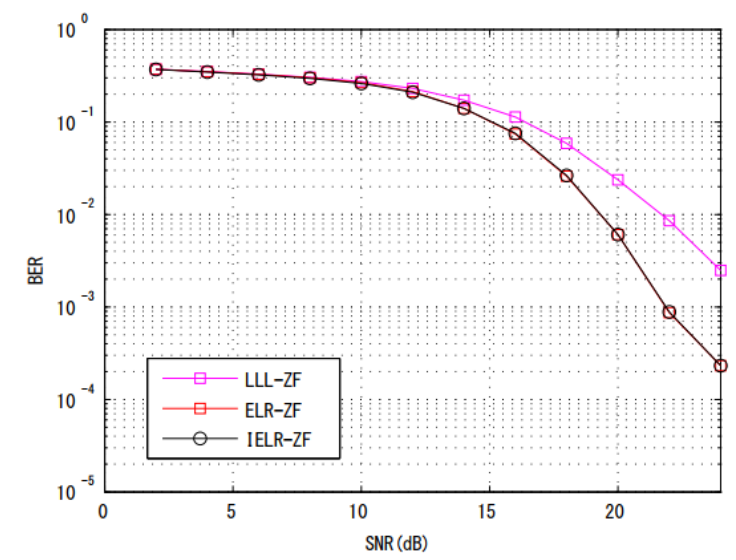

Fig. 1. Comparison of LLL-ZF, ELR-ZF and IELR-ZF for 16x16 MIMO.

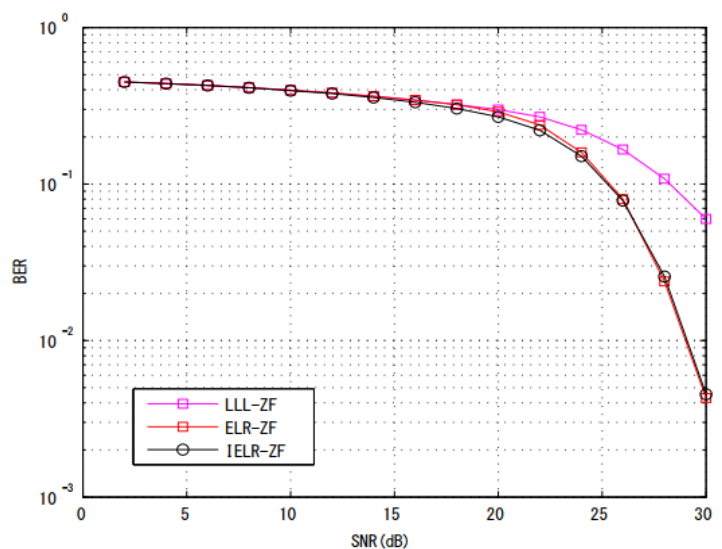

Fig. 2. Comparison of LLL-ZF, ELR-ZF and IELR-ZF for $64 \times 64$ MIMO.

Table 2. Assumption in Fig. 1 to Fig. 4.

\begin{tabular}{|l|l|l|l|}
\hline & TX antennas & RX antennas & Detection \\
\hline Fig. 1 & 16 & 16 & ZF \\
\hline Fig. 2 & 64 & 64 & ZF \\
\hline Fig. 3 & 16 & 16 & MMSE \\
\hline Fig. 4 & 64 & 64 & MMSE \\
\hline
\end{tabular}

axis represents BER (Bit Error Ratio). The assumption for the number of antennas and detection method are shown in Table 2. As you see, the BER performances of ELR and IELR are almost the same though the number of antennas and detection method are different.

\subsection{BER Performance via The Number of Antennas}

The BER performances of LLL, ELR, and IELR are shown in Fig. 5 and Fig. 6, all of them are in a condition that SNR is $30 \mathrm{~dB}$. ZF method is used for the simulation in Fig. 5 and MMSE method is used for the other. As you see from

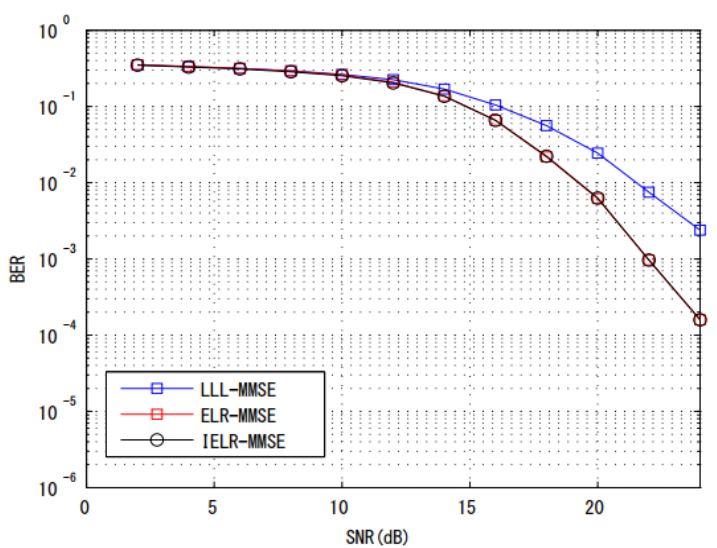

Fig. 3. Comparison of LLL-MMSE, ELR-MMSE, and IELR-MMSE for 16x16 MIMO.

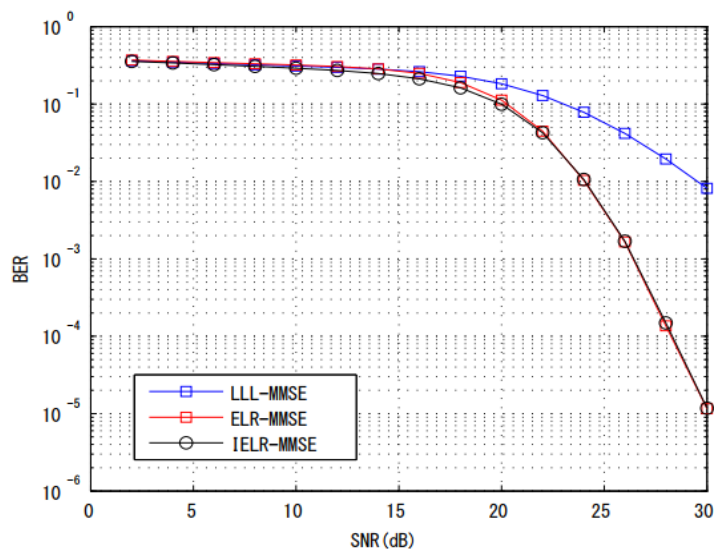

Fig. 4. Comparison of LLL-MMSE, ELR-MMSE and IELR-MMSE for 16x16 MIMO.

Fig. 5 and Fig. 6, the BER performance of ELR and IELR are almost the same, and they are superior to the BER of LLL.

\subsection{The Number of Iterations in The Original ELR Algorithm}

The number of iterations in the original ELR algorithm and ours are shown in Fig. 7. It shows the average number of iterations needed in LLL, ELR, and ours via the number of antennas. ELR needs the less iterations than LLL, and ours needs the least number of iterations in them. Moreover, as the number of antennas becomes large, the tendency becomes stronger.

The improvement ratios are shown in Table 3. IELR achieved about $20 \%$ improvement compared with ELR when the number of antennas is 30 , and the improvement ratio is over $40 \%$ when the number of antennas is 128 .

These results mean that IELR needs a lower iterations than ELR even though IELR and ELR are almost the same BER performances. 


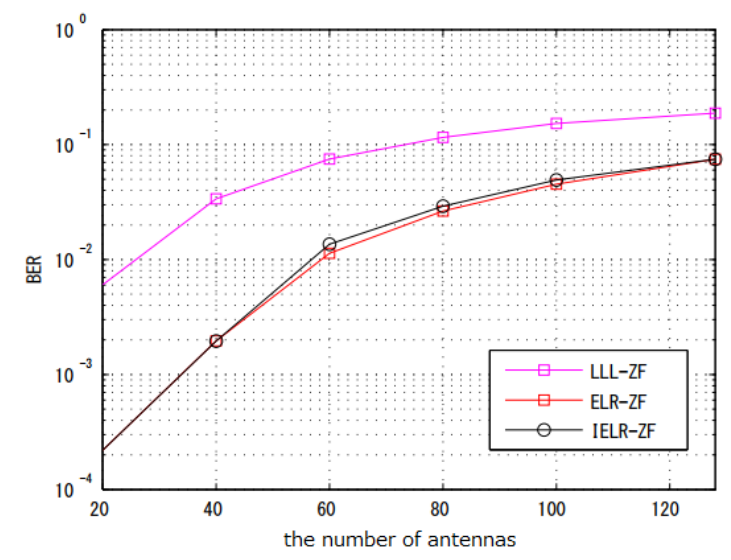

Fig. 5. Comparison of BER via the number of antennas using ZF.

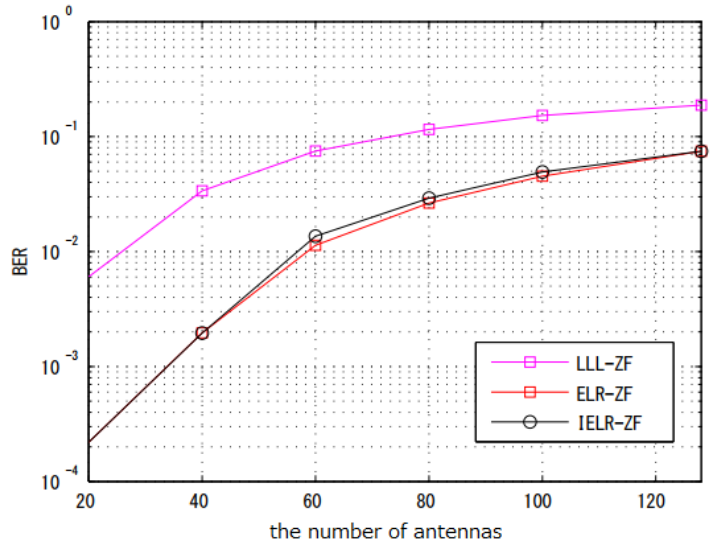

Fig. 6. Comparison of BER via the number of antennas using ZF.

Table 3. The improvement of IELR compared with ELR.

\begin{tabular}{|r|r|r|r|}
\hline \multirow{2}{*}{$\begin{array}{l}\text { TX \& RX } \\
\text { antennas }\end{array}$} & \multicolumn{2}{|l|}{$\begin{array}{l}\text { Average number of } \\
\text { iterations }\end{array}$} & \multicolumn{2}{l}{$\begin{array}{l}\text { Improvement } \\
{[\%]}\end{array}$} \\
\cline { 2 - 3 } & ELR & \multicolumn{1}{l|}{ IELR } & \\
\hline 10 & 19.18 & 17.81 & 7.143 \\
\hline 20 & 41.59 & 35.18 & 15.41 \\
\hline 30 & 63.07 & 49.51 & 21.50 \\
\hline 40 & 84.66 & 63.19 & 25.36 \\
\hline 50 & 106.2 & 76.34 & 28.12 \\
\hline 60 & 127.5 & 87.04 & 31.73 \\
\hline 70 & 148.8 & 99.54 & 33.10 \\
\hline 80 & 169.5 & 108.8 & 35.81 \\
\hline 90 & 192.9 & 122.4 & 36.55 \\
\hline 100 & 213.6 & 133.1 & 37.69 \\
\hline 128 & 271.8 & 160.5 & 40.95 \\
\hline & & & \\
\hline
\end{tabular}

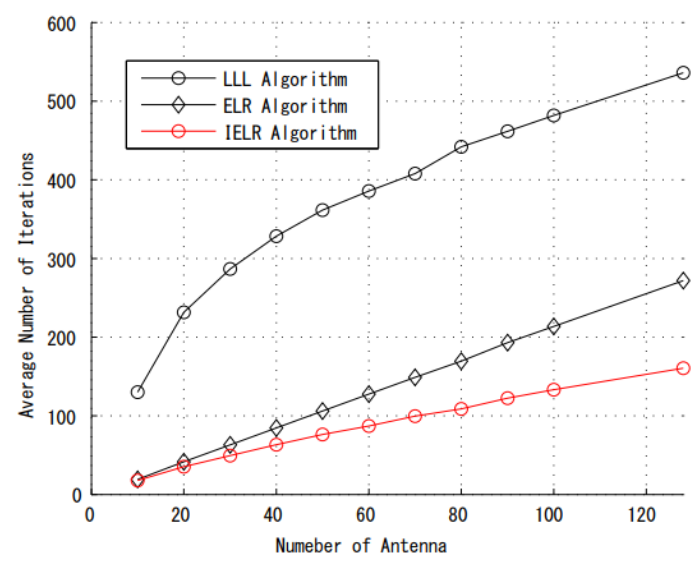

Fig. 7. Comparison of the number of iterations via the number of antennas.

\section{Conclusions}

The LR technique can obtain the diversity as high as the MLD method, but LR needs the low complexity than MLD. The ELR is one of LR, and the value of $\lambda_{i, k}$ which is used to update entries of some Matrixes in ELR algorithm. The number of iteration in ELR is big because the algorithm keeps calculation until all of $\lambda_{i, k}$ become zero for any $i$ except for $k$. We propose the new equation to calculate $\lambda_{i, k}$. The proposed $\lambda_{i, k}$ is consist of flooring function, and it can get to be zero easily compared to the original which is consist of rounding function. The orthogonality, however, becomes lower with our proposed value, but it doesn't matter because the orthogonality is enough to decrease PEP. We conducted three kinds of experiments. The first and the second experiments show that IELR ELR are superior to LLL in a view of BER and that IELR and ELR have almost the same BER performances. On the other hand, the third experiments shows that IELR needs a smaller number of iterations than ELR. Thus, it is cleared that our proposal can improve the original ELR.

\section{References}

(1) M. El-Hajjar and L. Hanzo, "Multifunctional MIMO systems: A combined diversity and multiplexing design perspective," IEEE Wireless Communications, vol.17 pp.73-79, April 2010.

(2) K. Zheng, L. Zhao, J. Mei, B. Shao, W. Xiang, and L. Hanzo, "Survey of large-scale MIMO systems," IEEE Communications Surveys Tutorials, vol.17, pp.17381760, third quarter 2015.

(3) L. Hanzo, M. El-Hajjar, and O. Alamri, "Near-capacity wireless transceivers and cooperative communications in the MIMO era: Evolution of standards, waveform 
design, and future perspectives," Proceedings of the IEEE, vol.99, pp.1343-1385, August 2011

(4) T. Marzetta, "Noncooperative cellular wireless with unlimited numbers of base station antennas," IEEE Transactions on Wireless Communications, vol.9, pp.3590-3600, November 2010.

(5) J. Zhang, B. Zhang, S. Chen, X. Mu, M. El-Hajjar, and L. Hanzo, "Pilot contamination elimination for largescale multiple-antenna aided OFDM systems," IEEE Journal of Selected Topics in signal Processing, vol.8, pp.759-772, October 2014.

(6) S. Yunchao, L. Chen, L. Feng, H.A. Zhao, “An improved detection algorithm based on Lattice reduction for MIMO system," Personal, Indoor, and Mobile Radio Communication, 214 IEEE 25th Annual International Symposium, pp.285-289, September 2014.

(7) A.K. Lenstra, H.W. Lenstra, and L.Lovasz, "Factoring polynomials with rational coefficients," Math. Annalen, vol.261, no.4, pp.515-534, 1982.

(8) X. Ma and W. Zhang, "Performance analysis for MIMO systems with lattice-reduction aided linear equalization," IEEE Transaction on Communications, vol.56, no.2, pp.309-318, February 2008.

(9) Y.H. Gan, C. Ling, and W.H. Mow, "Complex lattice reduction algorithm for low-complexity full-diversity MIMO detection," IEEE Transaction on Signal Processing, vol.57, no.7, pp.2701-2710, July 2009.

(10) M. Taherzadeh, A. Mobasher, and A. Khandani, "LLL reduction achives the receive diversity in MIMO decoding," IEEE Transaction on Information Theory, vol.53, no.12, pp.4801-4805, December 2007.

(11) Q. Zhou and X. Ma, "Element-based lattice reduction algorithms for large MIMO detection," IEEE Journal on Selected Areas in Communications, vol.31, pp.274-286, February 2013.

(12) W. Zhang, F. Arnold, and X. Ma, "An analysis of Seysen's lattice reduction algorithm," Signal Processing, vol.88, no.10, pp.2573-2577, May 2008.

(13) C.E. Chen and W.H. Sheen, "A new lattice reduction algorithm for LR-aided MIMO linear detection," IEEE Transaction on Wireless Communications, vol.10, no.99, pp.1-6, August 2011.

(14) Y. Song, C. Liu, F. Lu, "LR algorithm based on MED for multi-input-multi-output LDs," IET Communications, pp.1477-1487, June 2017. 